\title{
Germanium microparticles as optically induced oscillators in optical tweezers
}

\author{
W. H. Campos, ${ }^{1,2}$ T. A. Moura, ${ }^{1}$ O. J. B. J. Marques,${ }^{3}$ J. M. Fonseca, ${ }^{1}$ W. A. Moura-Melo, ${ }^{1}$ \\ M. S. Rocha, ${ }^{1}$ and J. B. S. Mendes $\oplus^{1, *}$ \\ ${ }^{1}$ Departamento de Física, Universidade Federal de Viçosa, 36570-900 Viçosa, Minas Gerais, Brazil \\ ${ }^{2}$ Institut für Physik, Johannes Gutenberg Universität Mainz, D-55128 Mainz, Germany \\ ${ }^{3}$ Programa de Pós-Graduação em Ciência de Materiais, Universidade Federal de Pernambuco, 50740-540 Recife, Pernambuco, Brazil
}

(Received 30 April 2019; published 21 November 2019)

\begin{abstract}
Oscillatory dynamics is a key tool in optical tweezer applications. It is usually implemented by mechanical interventions that cannot be optically controlled. In this paper, we show that germanium semiconductor beads behave as optically induced oscillators when subjected to a highly focused laser beam. In turn, the well-defined direction of oscillations can be manipulated by the polarization of the light beam. Such unusual motion is due to the competition between the usual optical forces and the radiometric force related to thermal effects, which pushes the beads from the focal region. We characterize the behavior of the germanium beads in detail and propose a model accounting for the related forces in good agreement with the experimental data. Such kind of system can potentially revolutionize the field of optical manipulation, contributing to the design of single molecule machines and to the application of oscillatory forces in fundamental physics, cellular manipulation, fluid dynamics, and other soft-matter systems.
\end{abstract}

DOI: 10.1103/PhysRevResearch.1.033119

\section{INTRODUCTION}

Optical tweezers (OTs) work by shining a highly focused laser beam onto small beads. Radiation pressure and radiometric forces push the particle away from the optical axis, while gradient forces, related to refraction effects, attract the object to the laser focus [1-4]. A metallic particle is usually pushed away, once light is mostly reflected and/or absorbed by the material. On the other hand, a dielectric microparticle suspended in water is usually observed to be trapped by the laser beam, since gradient forces overcome radiation pressure and radiometric forces [5-8]. OTs have applications in areas such as biochemistry, biophysics, microfluidics, colloidal sciences, and others, allowing mechanical studies of small soft-matter systems [5,9-16]. From an electric conducting point of view, semiconductor materials interpolate between dielectrics and metals, being the main ingredients for microelectronics technology. Consider shining light onto a semiconductor microparticle. What dynamics could we expect for it? Would it be trapped, like a dielectric, or drifted away, as occurs to metallic beads? To address these questions, we have conducted an experimental study upon the optical trapping and manipulation of germanium $(\mathrm{Ge})$ microspheres (diameter around a few micrometers) under the action of a highly focused Gaussian laser beam OT.

\footnotetext{
*Corresponding author: joaquim.mendes@ufv.br

Published by the American Physical Society under the terms of the Creative Commons Attribution 4.0 International license. Further distribution of this work must maintain attribution to the author(s) and the published article's title, journal citation, and DOI.
}

In turn, over 100 years after its discovery, Ge continues to attract tremendous attention in different areas of condensedmatter physics and materials science. Due to its suppression of spin relaxation, group IV semiconductors, such as Ge and $\mathrm{Si}$, have aroused interest in timely research branches, like spintronics [17-21]. Furthermore, compared with $\mathrm{Si}, \mathrm{Ge}$ possesses a much higher carrier mobility [20]. In this way, the solid-state device research community is returning to investigate the high-mobility Ge to improve the performance of transistors based on this material [21]. Nowadays, Ge has applications in optical fibers, polymerization catalysts, and Si-Ge alloys in microchip manufacturing, with feature sizes on the chips reaching $7 \mathrm{~nm}$ ( $<60 \mathrm{Ge}$ atoms $)$ [21,22]. However, to our best knowledge, the trapping of Ge microparticles in OTs has not been investigated so far.

Surprisingly, Ge beads are observed to oscillate in a plane perpendicular to the optical axis with relatively well-defined amplitude and frequency controllable by the laser power. Figure 1(a) shows our experimental setup, in which the particles are located around $z \sim-5 \mu \mathrm{m}$ below the focal plane. The position of a $3 \mu \mathrm{m}$-diameter Ge particle relative to the optical axis as function of time is shown in the inset, illustrating their typical oscillatory dynamics. In addition, we show that laser polarization can be used to easily guide the oscillations to a preferential direction, an appealing feature for practical applications. Such an oscillatory motion has the potential of extending usual OT setup capabilities for investigating dynamical properties of macromolecules and small systems, like DNA molecules and biological membranes.

Our paper is structured as follows. In Sec. II, we describe the syntheses and characterization of our Ge particles, the experimental setup of our OT, the experimental procedures and the data analysis to obtain the forces involved. In Sec. III, we present our experimental results and propose an effective 

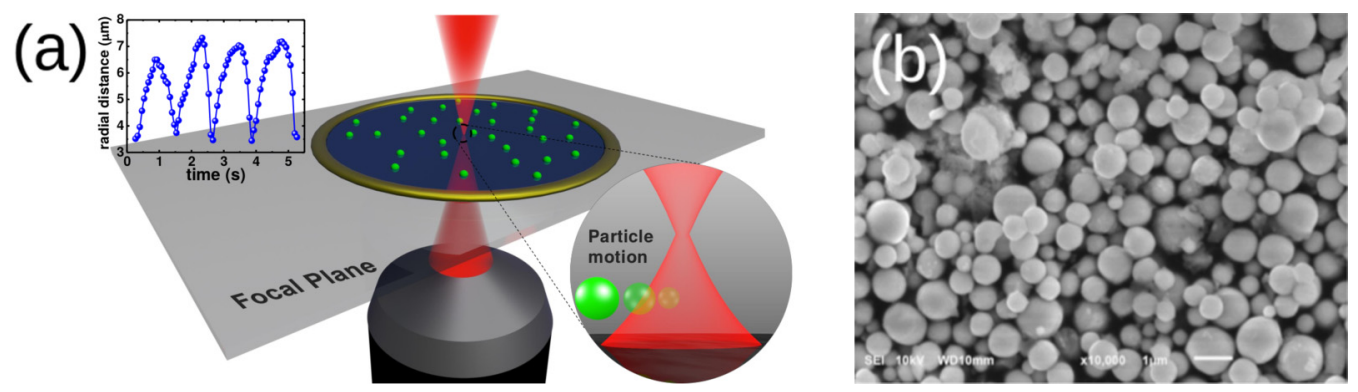

FIG. 1. (a) Experimental setup yielding the optically induced oscillations of Ge microspheres. The beads are suspended in deionized water and subjected to a Gaussian laser beam OT. The sample chamber consists of an o-ring glued in a microscope coverslip. We have observed that, after placing the beads in the sample chamber with deionized water, they remain suspended around $4 \mu \mathrm{m}$ above the coverslip surface, before and during the laser incidence. The inset shows the radial displacement of a bead, relative to the optical axis of the laser. (b) Scanning electron microscopy (SEM) of Ge microspheres obtained by the laser ablation technique. The particles present a well-defined spherical shape with smooth and homogeneous surface.

theoretical model to explain the observations. We also provide a comparison between theory and experiment and a discussion of the results. Finally, Sec. IV brings our conclusions and perspectives for future investigations.

\section{MATERIALS AND METHODS}

\section{A. Synthesis and characterization of the Ge microparticles}

The microparticles of Ge were synthesized by pulsed laser ablation technique in liquid solution. Pulsed laser ablation in liquids was carried out in a Nd:YAG laser Quantel, model Brilliant B. The laser light used for the ablation was the second harmonic wave $(\lambda=532 \mathrm{~nm})$ with a pulse energy of $70 \mathrm{~mJ}$ and $10 \mathrm{~Hz}$ in the nanosecond regime. The experimental setup used to synthesize the microparticles consists of using Ge crystals $(99,999 \%)$ as a target material immersed in $10 \mathrm{ml}$ of distilled water as a working liquid for ablation. The target was ultrasonically cleaned in distilled water before the process and placed at the bottom of a beaker. The laser beam was focused onto the Ge surface under a liquid layer of approximately $10 \mathrm{~mm}$ thick with a spot size of about $1 \mathrm{~mm}$ in diameter using focal lens of $50 \mathrm{~mm}$. The target was moved perpendicularly to the laser beam during 5 minutes to irradiate fresh surfaces during the whole process. The experiments were conducted at room temperature $(\sim 295 \mathrm{~K})$ and pressure of $1 \mathrm{~atm}$.

The high quality of the Ge microparticles was confirmed by scanning electron microscope [see Fig. 1(b)] and Raman spectroscopy analysis has proven that our Ge beads maintained their chemical structure during the laser ablation process (Fig. 2). Additional characterizations by energy dispersive $\mathrm{x}$-ray analysis have also demonstrated that the synthesized particles have no impurities in their composition.

\section{B. Optical tweezer setup}

The OT consists of a 1064-nm ytterbium-doped fiber laser (IPG Photonics) operating in the $\mathrm{TEM}_{00}$ mode, mounted on a Nikon Ti-S inverted microscope with a $100 \times$ NA 1.4 objective. We have set the laser power at $37 \mathrm{~mW}$, measured at the objective entrance. The sample chamber consists of an o-ring glued in a microscope coverslip.

\section{Experimental procedures and data analysis to obtain the forces}

For the optical experiment, we have selected those particles with well-defined spherical shapes. The individual motion of each Ge microparticle for various oscillation cycles was recorded using videomicroscopy. We have used a CCD camera (JAI BM-500GE) with a recording rate of 15 frames per second and a resolution of 29 pixels $/ \mu \mathrm{m}$. An exemplifying video showing the typical dynamics of a $3 \mu \mathrm{m}$ diameter $\mathrm{Ge}$ microsphere under linearly polarized Gaussian laser beam OTs can be found in the Supplemental Material [23]. Figure 3 shows successive frames of the video, along with a number of features of the particle oscillatory motion. From the video, it its evident that whenever a Ge particle is placed near the optical region, it is naturally impelled to oscillate in a preferential direction, i.e., perpendicular to the light polarization [Fig. 3(b)]. The video also shows that the Ge microparticles used in our experiments present a very good spherical shape, justifying the use of the laser ablation technique.

The videos were analyzed using ImageJ software, which allows us to obtain the coordinates of the particle centroid as a function of time. The optical axis position, $\left(x_{0}, y_{0}\right)$, can be

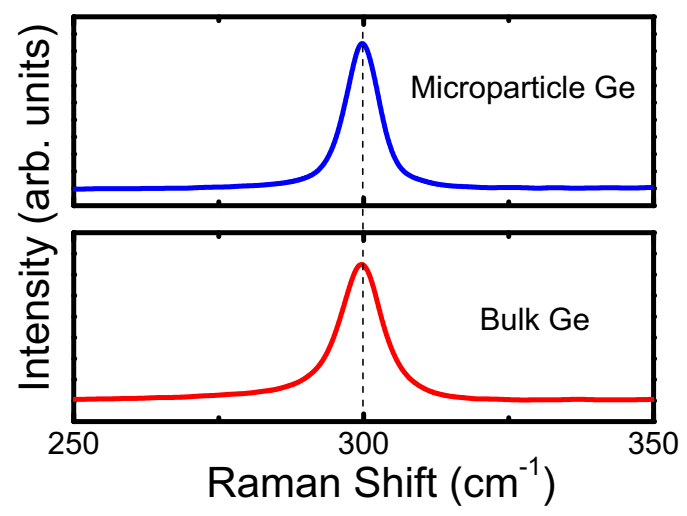

FIG. 2. Representative Raman spectra acquired from the Ge bulk (red color) and a Ge microparticle (blue color), highlighting the fundamental unstrained Ge Raman line at $\sim 300 \mathrm{~cm}^{-1}$, which corresponds to the bulk Ge phonon mode. The Raman spectra indicates that the particles did not experience chemical changes during the synthesis process. 
(a)
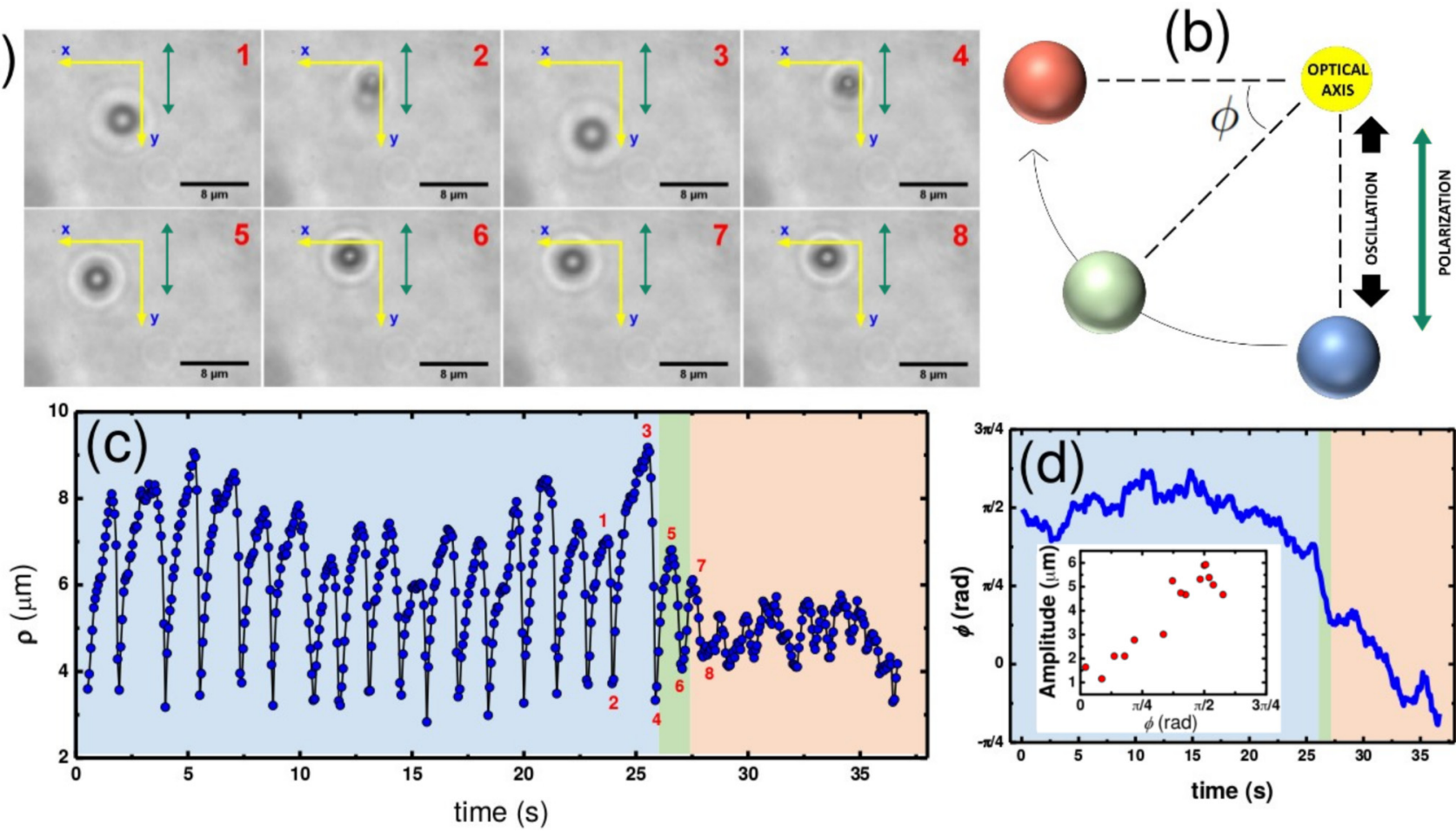

FIG. 3. Ge microparticle of $3.1 \mu \mathrm{m}$ diameter under a linearly polarized Gaussian laser beam OT. (a) Successive video frames showing the microsphere oscillating toward the optical axis (origin of the coordinate frame). (b) Direction of light polarization (green arrow), along with the definition of the azimuthal angle, $\phi$. (c) Radial displacement of the particle, $\rho$, as function of time. The blue (red) background indicates oscillations along the $y$ axis ( $x$ axis), while the green background points out the transient from one axis to the other. This typical oscillatory dynamics indicated by the numbers in red correspond to the frames shown in (a). (d) How the azimuthal angle, $\phi$, varies in time. The inset shows the amplitude of the oscillations versus $\phi$.

obtained by trapping a dielectric particle and taking the time average of its coordinates. Then, the radial position of the particle relative to the optical axis can be calculated by $\rho=$ $\sqrt{\left(x-x_{0}\right)^{2}+\left(y-y_{0}\right)^{2}}$. The velocity (acceleration) necessary to determine the Stokes (resultant) force is calculated by taking the first- (second-) order time derivative of the particle position. To compare the experimental results with the theoretical model accounting for radiometric and radiative forces, one has to subtract the contribution from the Stokes force,

$$
\vec{F}=\vec{F}_{\text {res }}-\vec{F}_{S},
$$

where $\vec{F}_{S}=-6 \pi \eta a \frac{d \vec{r}}{d t}$ and $\vec{F}_{\text {res }}=\frac{4}{3} \pi a^{3} \rho_{\mathrm{Ge}} \frac{d^{2} \vec{r}}{d t^{2}}$ are the Stokes and resultant forces, respectively. $\eta$ is the medium viscosity (we have used deionized water, $\eta=8.9 \times 10^{-4} \mathrm{Pas}$ ), $a$ is the particle radius and $\rho_{\mathrm{Ge}} \approx 5323 \mathrm{~kg} / \mathrm{m}^{3}$ is the Ge density.

The period of oscillation can be estimated by taking the time difference between two consecutive minimum (or maximum) displacements from the optical axis. In turn, the amplitude of oscillation is defined as the distance between the minimum and the maximum radial displacement of a given cycle.

\section{RESULTS AND DISCUSSIONS}

Let us define a cylindrical coordinate system whose origin is at the laser beam focus, say, $x_{0}=y_{0}=0$ [see Figs. 3(a) and 3(b)]. The $z$ axis is taken along the optical axis, pointing in the direction of the laser propagation. We take the $y$ axis along the polarization direction, and the $x$ axis defined by the right-hand rule. As usual, the position of the particle is represented by $\vec{r}=(\rho, \phi, z)$, where $\rho$ is the radial distance between the particle and the optical axis, while $\phi$ is the azimuthal angle, measured relative to the $x$ axis. How the particle radial distance, $\rho(x, y)=\sqrt{x^{2}+y^{2}}$, varies over time is plotted in Fig. 3(c). Notice that the particle is initially in a position parallel to the light polarization, say, along $y$ axis $(\phi=\pi / 2)$, where it begins to oscillate toward the optical axis. However, the oscillation direction changes with time, eventually reaching a direction perpendicular to the polarization axis, as shown in Fig. 3(d). Once there, the oscillatory direction is relatively stable around the $x$ axis $(\phi=0, \pi)$. The amplitude of oscillation appears to increase linearly with the azimuthal angle, $\phi$, in the range of $[0, \pi / 2$ ] [see inset of Fig. 3(d)]. In addition, the diffraction pattern of the microparticle changes periodically in time [see Video.avi [23] and Fig. 3(a)], indicating that it is also subject to oscillations along the direction of the laser propagation, a behavior predicted in Ref. [24].

\section{A. Theoretical model}

As briefly mentioned before, the oscillatory dynamics of semitransparent particles in OTs results from a delicate balance between optical (gradient force and radiation pressure) and radiometric forces [8]. The former is related to linear momentum transfer from the light beam to the particle and it has been extensively investigated in the last decades $[2,7,25-28]$. 
In turn, the radiometric force comes as a consequence of the temperature gradient induced on the surrounding medium, due to inhomogeneous heating of the particle, repelling it from the focal region. A satisfactory microscopic model describing this effect in a Gaussian laser beam OT is still lacking.

In Ref. [29], the authors extended the Mie-Debye spherical aberration theory to linearly polarized OTs. They have shown that linear polarization introduces strong axial asymmetry on the optical force due to Mie resonance effects. In other words, the components of the force acquire a sinusoidal dependence on $\phi$. These effects become particularly important when the lateral displacement is of the order of the particle radius. The components of the optical force in a linearly polarized Gaussian laser beam OT read [29]

$$
\begin{aligned}
& F_{j}(\rho, \phi, z)=\mathcal{F}_{j}^{\prime}(\rho, z)-\mathcal{F}_{j}^{\prime \prime}(\rho, z) \cos (2 \phi), \quad j=\rho, z \\
& F_{\phi}(\rho, \phi, z)=-\mathcal{F}_{\phi}(\rho, z) \sin (2 \phi) .
\end{aligned}
$$

The functions $\mathcal{F}_{j}^{\prime}$ and $\mathcal{F}_{j}^{\prime \prime}$ take a factor proportional to the laser intensity [29],

$$
I(\vec{r})=I_{0}(z) \exp \left(\frac{-2 \rho^{2}}{w(z)^{2}}\right),
$$

where $I_{0}(z)$ is the intensity at the optical axis, for a height $z$ above the focal plane. $w(z)=w_{0} \sqrt{1+\left(z / z_{R}\right)^{2}}$ is the beam waist for a given value of $z$, with $z_{R}=\pi w_{0}^{2} / \lambda$. $w_{0}$ is the beam waist at the focal plane, and $\lambda$ is the laser wavelength.

To incorporate these results into the effective model proposed in Ref. [8], $\mathcal{F}_{j}^{\prime}$ and $\mathcal{F}_{j}^{\prime \prime}(j=\rho, z)$ are taken proportional to $\partial_{j} I(\vec{r})$. Therefore, the radial component of the gradient force reads

$$
F_{G \rho}=-\frac{2 \rho \mathcal{F}_{G \rho} \exp (1 / 2)}{w(z)\left(1+\eta_{\rho}\right)} \exp \left(\frac{-2 \rho^{2}}{w(z)^{2}}\right)\left(1-\eta_{\rho} \cos 2 \phi\right) .
$$

$\mathcal{F}_{G \rho}$ is the maximum magnitude of $F_{G \rho}$ for a given value of $z$, which occurs at $\rho=w(z) / 2$ and $\phi= \pm \pi / 2 . \eta_{\rho}$ is a parameter that accounts for the relative strength of the asymmetric term. An analogous calculation for the $z$ component gives

$$
F_{G z}=\frac{2 e \mathcal{F}_{G z} \rho^{2}}{w(z)^{2}\left(1+\eta_{z}\right)} \exp \left(\frac{-2 \rho^{2}}{w(z)^{2}}\right)\left(1-\eta_{z} \cos 2 \phi\right) .
$$

The maximum magnitude of $F_{G z}, \mathcal{F}_{G z}$, occurs at $\rho=$ $w(z) / \sqrt{2}$ and $\phi= \pm \pi / 2$. $e$ is the Euler's number and $\eta_{z}$ accounts for the relative strength of the asymmetric term. Finally, Mie scattering theory also predicts a component of the force along $\hat{\phi}$ [29]:

$$
F_{G \phi}=-\frac{2 \rho \mathcal{F}_{G \phi} \exp (1 / 2)}{w(z)} \exp \left(\frac{-2 \rho^{2}}{w(z)^{2}}\right) \sin 2 \phi .
$$

$\mathcal{F}_{G \phi}$ is the maximum magnitude of $F_{G \phi}$, occurring at $\rho=$ $w(z) / 2$ and $\phi=n \pi / 4, n= \pm 1, \pm 3, \pm 5, \pm 7$.

The radiometric force is related to the temperature gradient caused by inhomogeneous heating of the particle and its surrounding medium. Such effect is in order due to the spatially varying intensity profile of the laser beam, which causes the hemisphere facing the optical axis to be heated more than the opposite side. Because heating is a consequence of light absorption, it is present whenever the extinction coefficient of the particle material is nonzero in the wavelength of the microscope ( $\lambda=1064 \mathrm{~nm}$, in our experiments), which is often the case for most metals and semiconductor materials $[2,8]$. Developing a microscopic model for the radiometric force is challenging, but for the purpose of this paper it suffices to consider it proportional to the intensity of the laser beam $[8,26]$ :

$$
\vec{F}_{R}=F_{R \rho} \hat{\rho}+F_{R z} \hat{z}=\left(\mathcal{F}_{R \rho} \hat{\rho}+\mathcal{F}_{R z} \hat{z}\right) \exp \left(\frac{-2 \rho^{2}}{w(z)^{2}}\right) .
$$

Notice that the parameters $\mathcal{F}_{G j}$ and $\mathcal{F}_{R j}$ are actually functions of $z$. In fact, $\mathcal{F}_{G z}$ changes its sign as one crosses the focal plane $\left(\mathcal{F}_{G z}>0\right.$ for $z<0$ and $\mathcal{F}_{G z}<0$ for $\left.z>0\right)$.

The resultant force exerted the resultant force exerted on the particle (after subtraction of the Stokes force), $\vec{F}=\vec{F}_{R}+$ $\vec{F}_{G}$, reads

$$
\begin{aligned}
\vec{F}= & \left\{\left[\mathcal{F}_{R \rho}-\frac{2 \rho \mathcal{F}_{G \rho} \exp (1 / 2)}{w(z)\left(1+\eta_{\rho}\right)}\left(1-\eta_{\rho} \cos 2 \phi\right)\right] \hat{\rho}\right. \\
& +\left[\mathcal{F}_{R z}+\frac{2 e \mathcal{F}_{G z} \rho^{2}}{w(z)^{2}\left(1+\eta_{z}\right)}\left(1-\eta_{z} \cos 2 \phi\right)\right] \hat{z} \\
& \left.-\frac{2 \rho \mathcal{F}_{G \phi} \exp (1 / 2)}{w(z)}(\sin 2 \phi) \hat{\phi}\right\} \exp \left(\frac{-2 \rho^{2}}{w(z)^{2}}\right)
\end{aligned}
$$

While the radiometric force and radiation pressure tend to push the particle away, gradient force works as a restoring action toward the laser beam focus. Depending on the optical properties of the material from which the particle is made, three main scenarios emerge: (1) Radiometric force dominates: As occurs to metallic beads, large absorption of the incident light results in inhomogeneous heating of the medium and the particles are drifted away. (2) Suitable conditions favoring optical trapping are accomplished: For example, a dielectric microsphere in which absorption and reflection of light are negligible and gradient force dominates. (3) A special situation takes place whenever both such forces have comparable magnitudes: The competition between them may yield oscillatory motion, as we have observed to occur with Ge microspheres. Actually, similar oscillations also happen with topological insulator $\mathrm{Bi}_{2} \mathrm{Te}_{3}$ and $\mathrm{Bi}_{2} \mathrm{Se}_{3}$ beads [8]. Once such a kind of compound shares some electrical and optical properties with semiconductors, we may wonder whether optically induced oscillatory motion is the typical dynamics of semiconductor beads under the action of focused laser light, as commonly used in OT setups.

\section{B. Comparison between theory and experiments}

We now proceed with the discussion of a very important aspect of this effective model. In the real experiments, the local temperature in all points of the system is constantly varying, heating up and cooling down as the particle oscillates. For example, as the particle approaches the optical axis, its temperature rapidly increases due to light absorption. When the radiometric force overcomes the gradient force, the particle is pushed away toward the outermost region of the beam. During its removal the particle cools down, as heat is transferred to the surrounding medium. Thermal equilibrium is achieved when the particle is far from the optical axis, then the cycle restarts. A precise description beyond our effective 

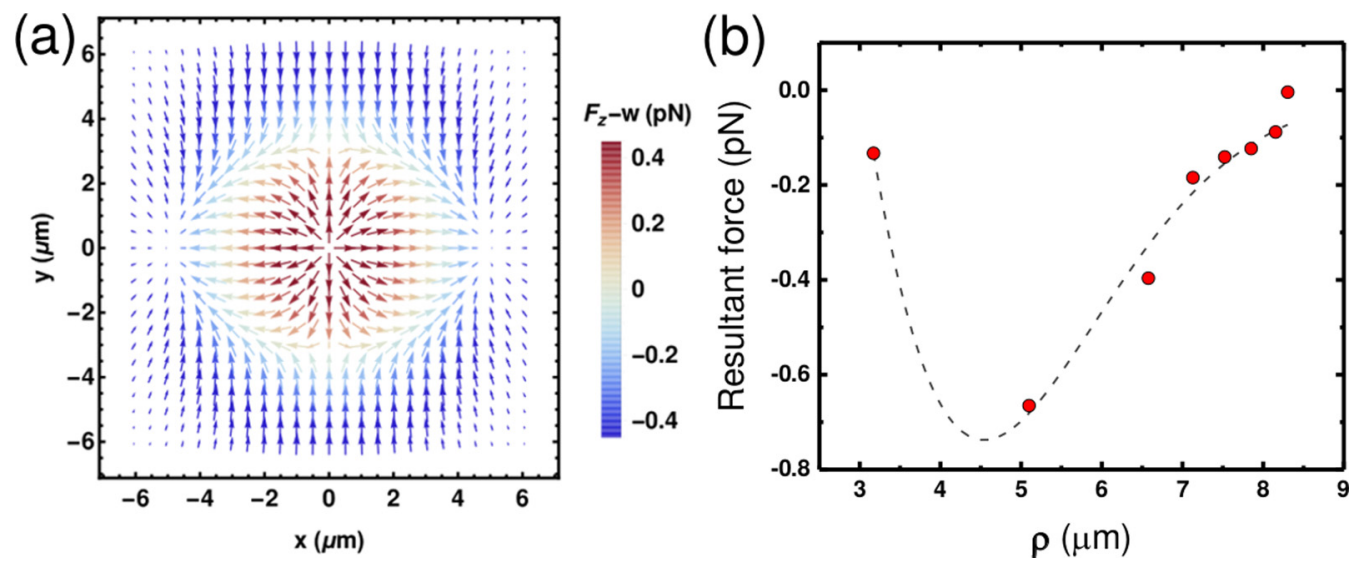

FIG. 4. (a) Typical vector plot of the resultant force, [Eq. (8)], in the approach regime. We have taken the following numeric values for the parameters: $w(z)=5.2 \mu \mathrm{m}, \mathcal{F}_{G \rho}=3.5 \mathrm{pN}, \mathcal{F}_{G \phi}=0.7 \mathrm{pN}, \eta_{\rho}=0.22, \mathcal{F}_{R \rho}=7.0 \mathrm{pN}, \mathcal{F}_{R z}=1.3 \mathrm{pN}$, and $\eta_{z}=0.1$. The in-plane vectors where plotted using the $x$ and $y$ components of the resultant force, while the color bar represents the total force along $z$ direction, $F_{z}-\mathrm{w}$, where $\overrightarrow{\mathrm{w}}=-(4 / 3) \pi a^{3}\left(\rho_{\mathrm{Ge}}-\rho_{m}\right) g \hat{z}$ is the apparent weight of the particle [24]. $F_{z}-\mathrm{w}>0$ for red vectors, while blue ones stand for $F_{z}-\mathrm{w}<0$. $a \approx 1.55 \mu \mathrm{m}$ is the sphere radius, $g=9.8 \mathrm{~m} / \mathrm{s}^{2}$ is the acceleration of gravity, $\rho_{\mathrm{Ge}}=5323 \mathrm{~kg} / \mathrm{m}^{3}$ and $\rho_{m}=997 \mathrm{~kg} / \mathrm{m}^{3}$ are the Ge and medium (deionized water) densities, respectively. (b) Fitting of the radial component of the resultant force, [Eq. (8)], to experimental data in the approach regime. Red dots are experimental data extracted from the third oscillatory cycle in Fig. 3(c), which takes place mostly along the polarization direction ( $y$ axis, in which the azimuthal component, $F_{\phi}$, is negligible). The dotted line is the fitting of the radial component of the resultant force with data from the approach regime. The fitting parameters along with their standard errors read as $\mathcal{F}_{R \rho}=(7.0 \pm 1.4) \mathrm{pN}$, $\mathcal{F}_{R g}=(3.59 \pm 0.56) \mathrm{pN}$, and $w(z)=(5.20 \pm 0.18) \mu \mathrm{m}$.

model is much more involved, once the physical parameters comprised in the resultant force, Eq. (8), are expected to bear complicated dependence on the microsphere and medium optical properties at each time instant. Therefore, as the forces depend on the refractive index, extinction coefficient, and other temperature sensitive properties, one should not expect the parameters of the model to be constant along all the oscillation cycle. An exception would be $w_{0}$, which is a characteristic of the laser beam [8]. To simplify our analysis, we can consider the effective values of the model parameters $\left(\mathcal{F}_{G \rho}, \mathcal{F}_{R \rho}\right.$, etc.) to be static, as long as we separate each cycle into two regimes: approach and removal.

Approach regime refers to the time interval in which the particle is moving toward the optical axis [e.g., from 1 to 2 in Fig. 3(c)]. The particle is initially at the outermost part of the optical region, in thermal equilibrium with the solution. The gradient force attracts the particle toward the optical axis and heating generates a radiometric force that opposes to the trapping. Figure 4(a) shows a vector plot of the resultant force in the approach regime, at a cross section of height $z=-0.5 \mu \mathrm{m}$ below the focal plane (see caption for parameters). The color bar represents the subtraction $F_{z}-w$, where $\vec{w}$ is the apparent weight of the particle. The vector plot shows red arrows for $F_{z}-w>0$ and blue arrows for $F_{z}-w<0$, which explains the small deviations from the oscillation plane that can be seen in the videos. The strong axial asymmetry induced by the polarization is evident, and the particle tends to oscillate in a preferential direction, i.e., the $x$ axis. Notice that there are two "stable" points along this direction, so the particle will be eventually attracted to one of them. However, for a particle initially placed at the $y$ axis, the time required for it to reach a stable point is longer than the oscillation period, so it performs a few oscillations before displacing to the $x$ axis. Along this direction, the amplitude of the oscillations decreases whenever compared to the $y$ axis (notice the smaller modulus of the resultant force vectors), by virtue of the polarization effect. Figure 4(b) shows the radial component of the resultant force, $F_{\rho}$, as function of the radial position, $\rho$, for the approach regime of the third oscillatory cycle in Fig. 3(c). The dashed line represents a theoretical fitting of $F_{\rho}$ [Eq. (8)] with experimental data. Among the fitting parameters, we have $w(z \approx$ $-5 \mu \mathrm{m})=(5.20 \pm 0.18) \mu \mathrm{m}$. From $w(z)$, the experimental parameter $w_{0-\exp }=(0.327 \pm 0.011) \mu \mathrm{m}$ is readily obtained, whose value is in good agreement with its theoretical counterpart $w_{0}=0.36 \mu \mathrm{m}$. [Taking the average over many cycles, we have obtained $\left.\left\langle w_{0-\exp }\right\rangle=(0.31 \pm 0.02) \mu \mathrm{m}\right]$. The good fitting and the small error bars suggest that the approximation of static parameters works well for the approach regime. As the particle moves toward the region of higher intensity, the temperature increases very fast around a particular distance to the optical axis. As a consequence, radiometric force overcomes the gradient and the transition to the removal regime takes place very abruptly, resulting sharp peaks in the particle position evolution at the points of minimum radial distance [ $\rho \sim 3 \mu \mathrm{m}$ in Fig. 3(c)].

Removal regime refers to the time interval in which the particle is moving away from the optical axis [e.g., from 2 to 3 in Fig. 3(c)]. The particle is initially at minimum radial distance and repulsive radiometric force dominates. Due to the high temperature gradient, the radiometric force is at its maximum, and repels the particle away from the optical axis. The radiometric force overcomes the gradient force along all the removal regime, until the particle reaches the outermost part of the optical region. Other effects can also contribute to repel the particle from the optical axis. We can cite for example the increase of radiation pressure due to particle heating, which changes the effective number of charge carriers 

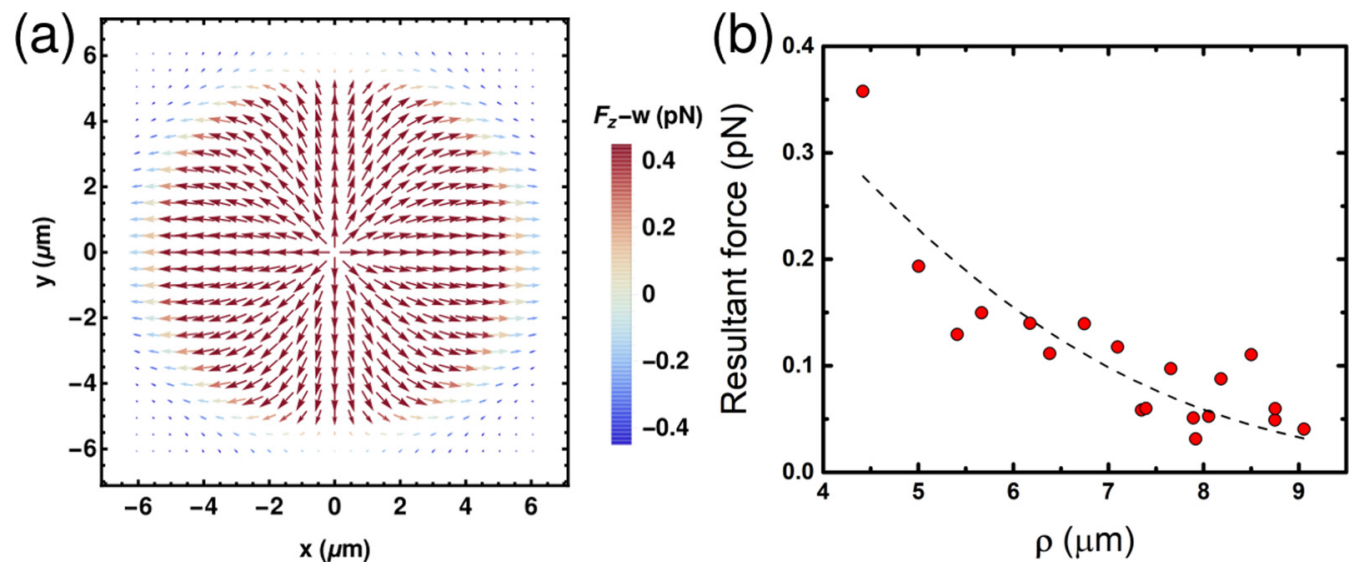

FIG. 5. (a) Typical vector plot of the resultant force [Eq. (8)] in the removal regime. The values of the parameters for the gradient force are the same as those used to generate Fig. 4(a). For the radiometric force, we have taken $\mathcal{F}_{R \rho}=13 \mathrm{pN}$ and $\mathcal{F}_{R z}=7.3 \mathrm{pN}$. It is clear from the plot that the radiometric force overcomes the gradient force along all removal of the particle, until it approaches the outermost part of the optical region and reaches thermal equilibrium with the surrounding medium. (b) Fitting of the radial component of the resultant force [Eq. (8)] to experimental data, in the removal regime. As the behavior of the experimental data points is much simpler than the general behavior of Eq. (8), the fitting return parameters with large error bars.

in the semiconductor particle, and a possible inversion in the direction of the gradient force that can occur due to changes on the dielectric constant of the particles, which occurs also due to heating [30,31]. At the end of this regime, the particle cools down until it reaches thermal equilibrium with the solution at the point of maximum distance. Thus, smooth transition to the approach regime takes place, restarting the cycle with the gradient force dominating the dynamics. Figure 5(a) shows a vector plot of the resultant force in the removal regime and Fig. 5(b) shows the fitting of the radial component of the resultant force, Eq. (8), to the experimental data for the removal regime. The oscillation cycle chosen to extract this data is the same as for Fig. 4(b). Although the model visually fits the experimental data, we must be attentive to the large error bars obtained from the fitting. The simpler behavior of the experimental data, compared to the general form of Eq. (8), results in too much freedom for the values extracted from the fitting. Therefore, the approximation of static parameters is not very accurate for the removal regime.

An animated image showing the dynamics of the resultant force field [Eq. (8)] is available in the Supplemental Material [23]. To generate the animation, we have manually set the values of $\mathcal{F}_{R \rho}$ and $\mathcal{F}_{R z}$ for each frame, while the parameters of the gradient force were kept constant [the same used to generate Fig. 4(a)]. Therefore, it should not be interpreted as a simulation, but rather as an illustration of the effective model.

We have also observed that fundamental control over period and amplitude of oscillations can be achieved by tuning laser power (see Fig. 6). These observations constitute a very robust experimental result, measured also for other similar particles (see, for example, Ref. [8]).

\section{Single-particle thermal machine}

We believe that the opto-hermal oscillations of $\mathrm{Ge}$ microparticles constitute a very rich scenario for the experimental realization of a single-particle thermal machine (microscopic engine) [32-34]. It has been recently reported the realization of a Brownian Carnot engine in which the working substance is a single polystyrene colloidal particle trapped in an OT [35]. However, important features make thermal machines with Ge particles a promising alternative. (1) Robust dynamics: It depends only on the optical properties of the particles and the medium. No external perturbation is necessary to achieve a thermodynamic cycle. (2) Injection of heat takes place via absorption of light, so the laser beam itself plays the role of the hot bath. A significant amount of energy that is usually wasted in conventional traps is converted into work in such system. (3) The large amplitude of oscillations is exceptionally appealing when it comes to practical applications of a thermal machine, such as exerting dynamic forces on polymers and other microscopic objects.

We may estimate the work done by cycle, $\mathcal{W}=\int_{\text {cycle }} \vec{F}$. $d \vec{l}$, and the efficiency, $\eta_{\text {eff }}$, of our Ge microsphere faced as an optomechanical engine. For that, let us recall that the forces $\vec{F}_{G}$ and $\vec{F}_{R}$ have components with magnitudes varying from

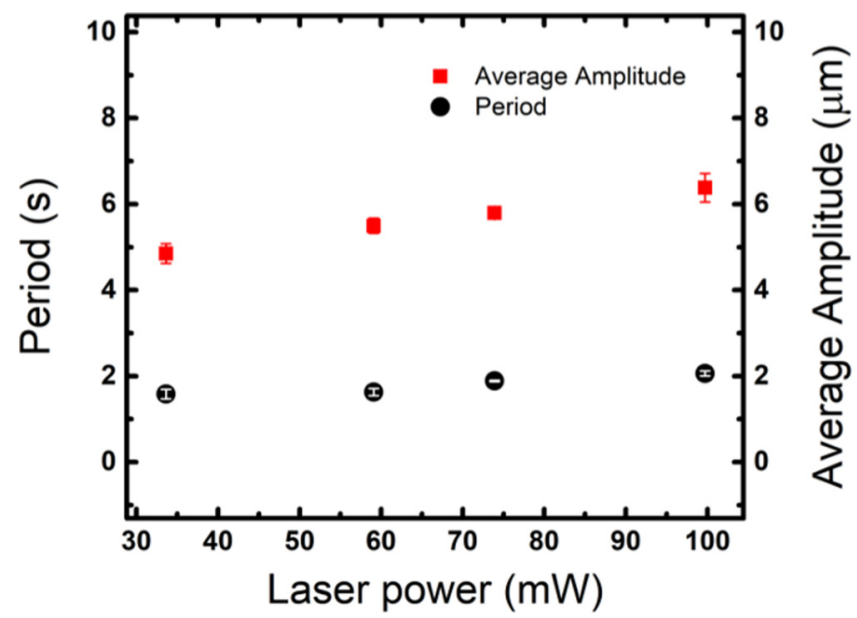

FIG. 6. Period (black dots) and amplitude (red squares) of oscillation for different laser power. Vertical bars are standard errors over averages. 
$\mathcal{F}_{G \phi}=0.7 \mathrm{pN}$ to $\mathcal{F}_{R \rho}=7.0 \mathrm{pN}$ (see caption from Fig. 4 for details). Larger amplitude particle oscillations, Figs. 3(c) and $3(\mathrm{~d})$, cover distances from $\rho_{\min } \approx 3 \mu \mathrm{m}$ to $\rho_{\max } \approx 9 \mu \mathrm{m}$, at frequencies $\sim 0.5-1 \mathrm{~Hz}$. In turn, shorter oscillations, taking place along the direction perpendicular to the laser polarization, go from $\rho_{\text {min }} \approx 3.5 \mu \mathrm{m}$ to $\rho_{\max } \approx 5.5 \mu \mathrm{m}$, with frequency $\sim 1-2 \mathrm{~Hz}$. Evaluating the integrals is lengthy but after some algebra we obtain

$$
\begin{aligned}
\mathcal{W} & \sim 10^{-18} \text { up to } 10^{-17} \mathrm{~J} \\
\Longrightarrow \quad \eta_{\text {eff }}=\frac{\mathcal{W}}{U_{\text {in }}} & \sim 10^{-15} \text { up to } 10^{-14},
\end{aligned}
$$

where the incident light energy onto the microsphere during a cycle reads $U_{\text {in }}=\int_{\text {cycle }} I_{o}(z) \pi a^{2} \exp \left(-2 \rho^{2} / w^{2}\right) d t \sim 2.5 \times$ $10^{-3} \mathrm{~J}$ (at $z \approx-5 \mu \mathrm{m}$ one has $w=w(z) \sim 5.2 \mu \mathrm{m}$ and $I_{0}(z) \approx$ $5 \times 10^{8} \mathrm{~W} / \mathrm{m}^{2}$ ). Specifically, the work done during the cycle presented in Fig. 4(b) gives $4.3 \times 10^{-18} \mathrm{~J}$. The performance of our $\mathrm{Ge}$ microsphere engine is comparable to that recently realized for a light absorbing microparticle whose dynamics comes about by demixing processes [36]. In this case, the estimated (maximum) work/cycle gives $373 k_{B} T(\sim 1.5 \times$ $10^{-18} \mathrm{~J}$ at room temperature) and efficiency of $1.16 \times 10^{-14}$. Such values are also comparable to those obtained for light absorbing microparticles propelled by explosive bubbles due to excess heating [37]. For instance, a $1-\mu \mathrm{m}$-size bead was reported to perform work/cycle $\sim 10^{-17} \mathrm{~J}$. Above performances are higher than those reported for colloidal heat Brownian-Carnot engine $\left(\mathcal{W}_{\max }=5 k_{B} T \approx 2.1 \times 10^{-20} \mathrm{~J}\right.$, at room temperature range) [35] and micrometer-scaled heat engine $\left(\mathcal{W}_{\max }=0.3 k_{B} T \approx 1.2 \times 10^{-21} \mathrm{~J}\right)$, see Ref. [38].

It is worth emphasizing that our proposal for a microscopic engine does not depend on mixing different specific liquids nor on boiling bubbles that interact with the beads (as it is the case for Refs. [36,37], respectively). The engine operates in the simplest possible medium and works at temperatures below the water boiling temperature. Furthermore, our work provides direct experimental verification of the results pointed out in Ref. [29], regarding the asymmetry in the optical force generated by linear polarization. Our approach suggests an application for semiconductors, opening an avenue of possibilities for the application of such materials in OTs and single molecule experiments.

\section{CONCLUSIONS}

In summary, Ge microparticles suspended in water are observed to oscillate whenever subject to a highly focused laser beam, with remarkable similarity to what occur to $\mathrm{Bi}_{2} \mathrm{Te}_{3}$ and $\mathrm{Bi}_{2} \mathrm{Se}_{3}$ topological insulator beads [8]. Once such kind of compound shares some electrical and optical properties with semiconductors, we may wonder whether optically induced oscillatory motion is the typical dynamics of semiconductor beads under the action of focused laser light. More specifically, Ge particles tend to oscillate in a direction perpendicular to the linear polarization of the laser beam. A possible explanation for such a dependence on the laser polarization is based on the theory of charge carriers in crystal lattice materials: Ge has a considerable density of free carriers inside its bulk, rendering a strong response to the light polarization. In contrast, topological insulators compounds have most of their free electrons lying on the surface, due to the energy band gap in the bulk of the material. This would justify why the topological insulator beads are practically insensitive to light polarization, as reported in Ref. [8]. Our present findings suggest that semiconductor-type beads tend to oscillate under the action of highly focused laser beams, as those used in OT setups. In fact, we also verified the signature of this behavior for Si microparticles, which indicates that such a phenomenon may be typical of conventional semiconductor materials. Therefore, these materials could also find application for dynamical measurements of mechanical properties of small systems, including macromolecules such as DNA, membranes, and so forth.

\section{ACKNOWLEDGMENTS}

The authors are grateful to Marcelo L. Martins for insightful discussions regarding single-particle thermal machines and Libor Šmejkal for reviewing the paper. This study was financed in part by the Coordenação de Aperfeiçoamento de Pessoal de Nível Superior - Brazil (CAPES), Finance Code 001. The authors also thank the Brazilian agencies: Conselho Nacional de Desenvolvimento Científico e Tecnológico (CNPq), Financiadora de Estudos e Projetos (FINEP), and Fundação de Amparo à Pesquisa do Estado de Minas Gerais (FAPEMIG) for financial support.
[1] A. Ashkin, Phys. Rev. Lett. 24, 156 (1970).

[2] A. Ashkin, Biophys. J. 61, 569 (1992).

[3] A. Ashkin, Nat. Photonics 5, 316 (2011).

[4] M. A. Taylor, M. Waleed, A. B. Stilgoe, H. RubinszteinDunlop, and W. P. Bowen, Nat. Photonics 9, 669 (2015).

[5] K. Svoboda and S. M. Block, Annu. Rev. Biophys. Biomol. Struct. 23, 247 (1994).

[6] K. Svoboda and S. M. Block, Opt. Lett. 19, 930 (1994).

[7] M. S. Rocha, Am. J. Phys. 77, 704 (2009).

[8] W. H. Campos, J. M. Fonseca, V. E. Carvalho, J. B. S. Mendes, M. S. Rocha, and W. A. Moura-Melo, ACS Photonics 5, 741 (2018)

[9] A. Ashkin and J. M. Dziedzic, Science 235, 1517 (1987).
[10] D. G. Grier, Nature 424, 810 (2003).

[11] K. C. Neuman and S. M. Block, Rev. Sci. Instrum. 75, 2787 (2004).

[12] J. R. Moffitt, Y. R. Chemla, S. B. Smith, and C. Bustamante, Annu. Rev. Biochem. 77, 205 (2008).

[13] K. C. Neuman and A. Nagy, Nat. Methods 5, 491 (2008).

[14] H. Kress, J.-G. Park, C. O. Mejean, J. D. Forster, J. Park, S. S. Walse, Y. Zhang, D. Wu, O. D. Weiner, T. M. Fahmy et al., Nat. Methods 6, 905 (2009).

[15] F. M. Fazal and S. M. Block, Nat. Photonics 5, 318 (2011).

[16] M. Capitanio, M. Canepari, M. Maffei, D. Beneventi, C. Monico, F. Vanzi, R. Bottinelli, and F. S. Pavone, Nat. Methods 9, 1013 (2012). 
[17] F. Pezzoli, F. Bottegoni, D. Trivedi, F. Ciccacci, A. Giorgioni, P. Li, S. Cecchi, E. Grilli, Y. Song, M. Guzzi, H. Dery, and G. Isella, Phys. Rev. Lett. 108, 156603 (2012).

[18] S. Dushenko, M. Koike, Y. Ando, T. Shinjo, M. Myronov, and M. Shiraishi, Phys. Rev. Lett. 114, 196602 (2015).

[19] F. Bottegoni, C. Zucchetti, S. Dal Conte, J. Frigerio, E. Carpene, C. Vergnaud, M. Jamet, G. Isella, F. Ciccacci, G. Cerullo, and M. Finazzi, Phys. Rev. Lett. 118, 167402 (2017).

[20] S. M. Sze and K. K. Ng, Physics of Semiconductor Devices, 2nd ed. (Wiley-Interscience, New York, 1981).

[21] R. Pillarisetty, Nature 479, 324 (2011).

[22] S. C. Burdette and B. F. Thornton, Nat. Chem. 10, 244 (2018).

[23] See Supplemental Material at http://link.aps.org/supplemental/ 10.1103/PhysRevResearch.1.033119 for an exemplifying video showing the typical dynamics of a $3 \mu \mathrm{m}$ diameter Gemicrosphere under our linearly polarized Gaussian laser beam optical tweezers (Video.avi) and an animated image showing the dynamics of the resultant force field [Eq. (8)] (AnimatedImage.gif).

[24] W. H. Campos, J. M. Fonseca, J. B. S. Mendes, M. S. Rocha, and W. A. Moura-Melo, Appl. Opt. 57, 7216 (2018).

[25] R. C. Gauthier and S. Wallace, J. Opt. Soc. Am. B 12, 1680 (1995).
[26] J. P. Gordon, Phys. Rev. A 8, 14 (1973).

[27] A. Mazolli, P. A. Maia Neto, and H. M. Nussenzveig, Proc. R. Soc. London A 459, 3021 (2003).

[28] R. S. Dutra, N. B. Viana, P. A. Maia Neto, and H. M. Nussenzveig, Appl. Phys. Lett. 100, 131115 (2012).

[29] R. S. Dutra, N. B. Viana, P. A. M. Neto, and H. M. Nussenzveig, J. Opt. A: Pure Appl. Opt. 9, S221 (2007).

[30] P. C. Ke and M. Gu, Appl. Opt. 38, 160 (1999).

[31] A. Lehmuskero, P. Johansson, H. Rubinsztein-Dunlop, L. Tong, and M. Kall, ACS Nano 9, 3453 (2015).

[32] L. A. C. A. Defaveri, W. A. M. Morgado, and Silvio M. Duarte Queirós, Phys. Rev. E 96, 052115 (2017).

[33] L. A. C. A. Defaveri, Silvio M. Duarte Queirós, and W. A. M. Morgado, Phys. Rev. E 98, 062106 (2018).

[34] I. A. Martinez, É. Roldán, L. Dinis, and R. A. Rica, Soft Matter 13, 22 (2017).

[35] I. A. Martínez, E. Roldán, L. Dinis, D. Petrov, J. M. R. Parrondo, and R. A. Rica, Nat. Phys. 12, 67 (2016).

[36] F. Schmidt, A. Magazzù, A. Callegari, L. Biancofiore, F. Cichos, and G. Volpe, Phys. Rev. Lett. 120, 068004 (2018).

[37] P. A. Quinto-Su, Nat. Commun. 5, 5889 (2014).

[38] V. Blickle and C. Bechinger, Nat. Phys. 8, 143 (2012). 